\title{
The Dynamic Control of the Light Signalling Device in Real-Time
}

\author{
Jan Mrazek ${ }^{1}$, Lucia Duricova Mrazkova $^{2}$, Martin Hromada ${ }^{3}$ and Jana Reznickova ${ }^{4}$ \\ ${ }^{1,2,3,4}$ Tomas Bata University in Zlin, Faculty of Applied Informatics, Nad Stranemi 4511, Zlin, 760 05, Czec Republic
}

\begin{abstract}
The article is focused on the issue of interval on a light signaling device. Light signaling devices operate on different systems by means of which they are controlled. The control problem is a very static setting that does not respond to real-time traffic. Important variables for dynamic real-time control are traffic density in a selected area along with average speed. These variables are interdependent and can be based on dynamic traffic control. Dynamic traffic control ensures smoother traffic through major turns. At the same time, the number of harmful $\mathrm{CO} 2$ emitted from the means of transport should be reduced to the air. When used in low operation, power consumption should be reduced.
\end{abstract}

\section{Introduction}

Critical infrastructure is an important issue in every country. Its proper functioning and safe status are priorities for each stat. Elements of critical infrastructure include the transport sector.

Transport is divided into four sectors. These sectors include rail, road, air and water transport. The most popular and most used are rail and road transport. Road transport is at the top of the popularity of transport sectors. These facts are confirmed in recent years.

Roads are most risky because of their popularity. The amount of people and material that is transported annually increases the risk of a crisis. Subsequently, a crisis situation may eventually lead to an emergency.

Currently, the failure of light signaling devices, traffic accidents, closures, etc. can be categorized as a crisis situation.

Light signaling devices are already taken for granted. A common outage creates a great deal of nervousness and a high risk of traffic accidents due to inattention or poor response. For this reason, it is appropriate to address the issue of driving a signaling device that can provide safety. In the most popular times, colony formation can occur that creates nervousness and other negative traits among drivers.

The available methods used in real-time control of the light signaling device but no further aspects are being processed. These aspects include traffic density and average speed. These aspects are directly related to one another.

The proposed dynamic control method works directly with these aspects. Real data is processed in real time to make traffic management efficient.

The conclusion of the paper is a case study that describes the way of dynamic control of the light signaling device in real time. The case study describes the management stages and the differences between the current situation.
At the end of the article, the description of the preferred light control device preferred solution is preferred.

\section{Critical infrastructure}

Critical infrastructure is a system that is necessary to ensure the protection of the lives and health of people and property. Systems include physical, cyber and organizational.

Important elements that should not be influenced by functionality and poor include sectoral criteria that need to be protected. For every country, the correct functionality and safety of the industry are important.[1]

\subsection{Cross-cutting criteria}

The cross-sectional criterion for determining a critical infrastructure element is:

-Victims with a threshold of more than 250 deaths or more than 2500 people with subsequent hospitalization for more than 24 hours

- an economic impact with a marginal economic loss of more than $0.5 \%$ of gross domestic product;

-Impact on the public with a large-scale limitation on the provision of essential services or other serious interference to everyday life affecting more than 125,000 people.

\subsection{Sectoral criteria}

Used to identify a critical infrastructure element. In addition, the sectors listed below are listed below:

- Energy

- Water management

- Food and Agriculture

\footnotetext{
* Corresponding author: jmrazek@utb.cz
} 
- Healthcare

- Traffic

- Communication and information systems

- Financial market and currency

- Emergency services

- Public administration.

In the next section, we will focus on the transport sector to which

we will deal with the rest of the article and solve the problems of control by means of light signaling devices.

\section{Means of transport in the Czech Republic}

The fleet in the Czech Republic is obsolete and dangerous for a long time. The number of means of transport is increasing every year. The popularity of road transport is confirmed by the numbers of registered cars in recent years. In Table 1 below we can see the numbers of registered means of transport divided into categories. [4]

Table 1 Number of means of transport by category. [2]

\begin{tabular}{|c|c|c|}
\hline & $\mathbf{2 0 1 6}$ & $\mathbf{2 0 1 7}$ \\
\hline Motorcycles & $\begin{array}{c}1074 \\
880\end{array}$ & 1102392 \\
\hline Cars & $\begin{array}{c}5307 \\
808\end{array}$ & 5538222 \\
\hline Bus & 20097 & 20719 \\
\hline Trucks & 667705 & 689368 \\
\hline $\begin{array}{c}\text { Road } \\
\text { tractors }\end{array}$ & 4488 & 4132 \\
\hline Semitrailers & 53826 & 52855 \\
\hline Trailers & 423373 & 434872 \\
\hline Special cars & 31886 & 31277 \\
\hline TOTAL & $\mathbf{7 5 8 4}$ & $\mathbf{7 8 7 3 8 3 7}$ \\
& $\mathbf{0 6 3}$ & \\
\hline
\end{tabular}

In the annual census, we can observe this increase. This trend is related to the age of the fleet. Together with the increase in the number of individual categories, the age in each category is increasing. [4]

At the end of 2016, the average car age was 14.48 years on the car. In terms of age, we need to work on dynamic management to minimize the release of harmful substances from these categories. Fluency not only reduces these values but also reduces power consumption and increases safety. The increase in safety should be due to ensuring continuity on the main roads in order to minimize the possibility of traffic accidents in the columns. [2]

\section{Current Trends in the Control of Light Signaling Devices}

Light signaling devices are used to control roads in places that are very dangerous without their use or to ensure smooth traffic. Current trends are mostly static. The dynamic part occurs only in the case of lightsignaling control within the priorities for urban public transport or integrated rescue system components.

Dynamic driving should be a reality at this time and not a sci-fi. Now we will be familiar with current trends in light control. [9], [10], [11]

\subsection{Fixed signaling plane}

The fixed signaling plan is characterized by a static function, when exactly defining the time intervals of the signal changes from one instruction to the next and how many directions the individual directions will have or will be. [6], [7]

The design of fixed signaling plans consists of the following steps:

- Phase diagram assembly.

- Split times calculation.

- Cycle length calculation.

- Calculation of individual cycle times.

\subsection{Variable signaling plane}

The variable signaling plan is most often used depending on the most popular times. These times can be defined as times when people commute to work or school and vice versa. These variable signaling plans have some significance but do not work on the dynamic part depending on traffic density. [6], [7]

\subsection{Video-detection}

The control of the light signaling device by means of video detection can already be characterized as dynamically controlled intersections. A big drawback when controlling video detection is the distance to which cars can be detected. It works to fill the zone when the signal is switched off. In part, video-detection can be referred to as a dynamic element but very restrictive in terms of distance it monitors. [6], [7]

\subsection{Induction loop detection}

Induction loops are located in the roadways. A great advantage is an instruction to drive a car to give preference. The induction loops can be applied mostly to places where there is not a large number of cars passing through, but rather to places where there is a frequent change of directions and at the same time, there is a medium traffic density. [6], [7]

\section{Traffic Control Case Study}

In the case study, we will focus on controlling the light signaling device. The light signaling device is an 
element that we meet every day not only as a driver but also as a pedestrian. This system can be characterized as a control system, facilitating the movement of drivers and pedestrians on roads, sidewalks or in places where it is necessary to crossroads.

Our solution for driving a light-signaling device is based on dynamic control. Quantities affecting our proposed traffic management solution are traffic density and average speed.

In Figure 1 we can see an example in which we will further explain the principle of control.

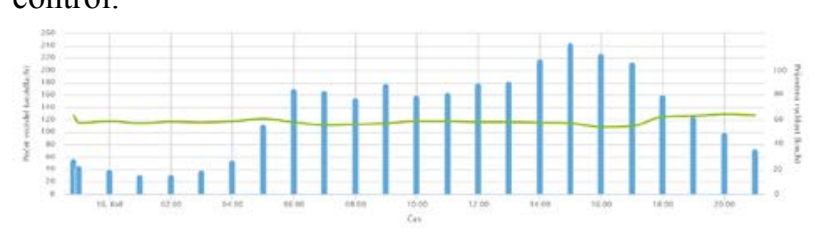

\section{Figure 1 Example of data for dynamic control.}

The blue color indicates the number of vehicles in each hour. The green line is the average speed in each hour along with the number of vehicles. This curve also shows us the times when the vehicles slowed down due to a crisis situation. There are several reasons for a situation that has occurred. These causes include:

- Traffic accident,

-road reconstruction,

- traffic restrictions,

-other.

Different limits for acceptable states can be set based on the calculation of the maximum permeability of vehicles in the measured section.

\subsection{Out of service light}

The light signaling device will ensure the saving of electric energy together with the minimization of harmful substances of $\mathrm{CO} 2$. This state is set when the average number of vehicles does not exceed 45 vehicles per hour. This condition will most often occur at night and weekends when traffic density is poor. With low traffic density, we can improve the air but I reduce the energy consumption of the light signaling device. This condition can be applied to a larger number of light signaling devices.

Each city has, within its security co-ordinator, selected locations or sections that are dangerous to a crisis situation. These places are equipped with a light signaling device which cannot be switched off in order to avoid endangering the safety of the population and traffic participants.
In Figure 2, a red line marked the acceptable limit. This limit indicates the limit which, when exceeded, instructs the light signaling device to start. The activation of the light-signaling device must be at the lower limit in order to increase safety. This value is different and it depends on which section the system will be applied to. Some roads may have a value of about 45 vehicles per hour and others may have this lower limit set up to about 140 vehicles per hour.

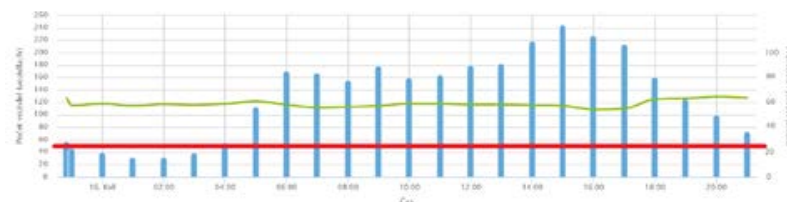

\section{Figure 2 Setting the upper limit to trigger the light signaling device.}

\subsection{Traffic lights in operation}

Traffic signaling devices in operation where different road junctions are common. In our case, we set different levels of control, as shown in Figure 3. These levels can be set to any number. Levels can be more or less as needed.

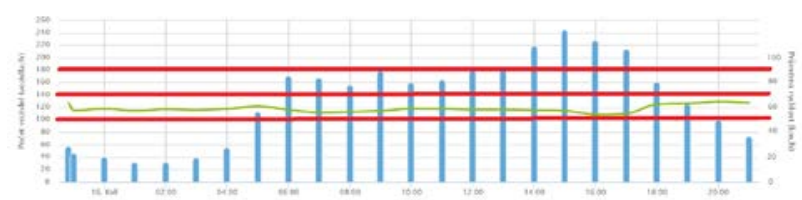

Figure 3 Set limit values to adjust the signal free.

Figure 3 shows three different levels that are marked with red lines in the image. Each line indicates a level that instructs the controller when it exceeds the number of cars. After receiving these pulses, the controller extends or shortens the length of the green intervals.

When the first level is exceeded, the signaling device in the signal direction increases by 20 seconds. This value can be set as needed and space. All data can be set to the location as needed. It can be stated that after minor testing it is possible to set the system in the given locality.

When the second level is exceeded, the signaling device will increase for the next 20 seconds in the signaling direction. This phenomenon is repeated according to the number of levels we have set. The number of levels can be set to more or less as needed.

Each level also works at the average speed. Measurements are only made by informative measurement, which records the speed of passing vehicles and then averages them. The speed average can show us in real-time the problem in a section that we can respond to using the proposed method in real-time.

When comparing the density and average speed, it is possible to set values that occur in the event of a traffic jam or a traffic accident. These events most affect the 
average speed of vehicles and thus should occur to minimize errors when setting the interval.

\section{Conclusion}

Critical infrastructure elements can be seen as systems. Each system is designed to function properly and security is taken into account. Security both internally and externally. A secure system ensures well-being and peace of mind is essential to ensuring that critical infrastructure industries are functioning and safe.

Transport is one of the branches of critical infrastructure elements. Traffic is divided into four subcategories. Sub categories include road, rail, water, and air.

Road transport is seen as the most popular form of transport, but also the most dangerous from the point of view of popularity I. It is predominantly dangerous from the point of view of the amount of material and people transported by road. The number of means of transport is increasing as a result of the amount that is transported on the roads every day This increases the risk of road accidents.

The light signaling device serves as a security element that controls the intersection of roads and ensures safety and fluency in operation based on preset intervals or by controllers. A controller is an element that can be called the brain of a light-signaling device.

The light signaling control solution we propose is based on dynamic traffic control. Based on the predefined lower and upper limits, acceptable limits are set that should change the signal length in each direction. The proposed solution should ensure power saving while the power is off. With the traffic light on, it should predominantly ensure the reduction of harmful substances $\mathrm{CO} 2$, fluency, safety and permeability on priority sections, especially in dense traffic, to avoid colonies. Among the disadvantages of the solution is to sort the settings between each section.

The greatest advantage of the proposed system is dynamic control. The entire control is done in real-time. Reducing the interval can be set as needed. Effective reduction of the time interval appears to be most appropriate with an hourly interval and with the evaluation of traffic density and average speed in a given measured section.

\section{Acknowledgments}

This project is realized as the research with doctoral student and it is the basic input for next research, which we will develop in next term. It was realized with support of the university. This work was supported by Internal Grant Agency of Tomas Bata University under the project No. IGA/FAI/2019/010.
This work was supported by the research project VI20152019049 "RESILIENCE 2015: Dynamic Resilience Evaluation of Interrelated Critical Infrastructure Subsystems", supported by the Ministry of the Interior of the Czech Republic in the years 20152019.

\section{References}

1. MRAZEK, Jan, DURICOVA, Lucia \& HROMADA, Martin. The Proposal of Evaluation Criteria for Recoverability of Road Transport. Safety and Reliability - Theory and Applications Čepins \& Briš (Eds), 2017. Taylor \& Francis Group, London. ISBN 978-1-138-62937-0.

2. Czech Statistical Office. Statistical yearbook of the Czech republic 2018. Czech statistical office 2018. From https://www.czso.cz.

3. ZHANG, Linfeng, et al. Deep potential molecular dynamics: a scalable model with the accuracy of quantum mechanics. Physical review letters, 2018, 120.14: 143001.

4. EHSANI, Mehrsa; AHMADI, Abbas; FADAI, Dawud. Modeling of vehicle fuel consumption and carbon dioxide emission in road transport. Renewable and sustainable energy reviews, 2016, 53: 1638-1648.

5. SOYSAL, Mehmet; ÇIMEN, Mustafa; DEMIR, Emrah. On the mathematical modeling of green oneto-one pickup and delivery problem with road segmentation. Journal of cleaner production, 2018, 174: 1664-1678.

6. CHIOU, Suh-Wen. A traffic-responsive signal control to enhance road network resilience with hazmat transportation in multiple periods. Reliability Engineering \& System Safety, 2018, 175: 105-118.

7. KEIMER, Alexander, et al. Information patterns in the modeling and design of mobility management services. Proceedings of the IEEE, 2018, 106.4: 554-576."

8. LIU, Jian, et al. Secure intelligent traffic light control using fog computing. Future Generation Computer Systems, 2018, 78: 817-824.

9. WEI, Hua, et al. Intellilight: A reinforcement learning approach for intelligent traffic light control. In: Proceedings of the 24th ACM SIGKDD International Conference on Knowledge Discovery \& Data Mining. ACM, 2018. p. 2496-2505.

10. NILSSON, Gustav; COMO, Giacomo. Evaluation of decentralized feedback traffic light control with dynamic cycle length. IFAC-PapersOnLine, 2018, 51.9: 464-469. 
11. SUN, Chao, et al. Robust eco-driving control of autonomous vehicles connected to traffic lights. arXiv preprint arXiv:1802.05815, 2018.

12. ZHOU, Binbin, et al. Adaptive traffic light control in wireless sensor network-based intelligent transportation system. In: 2010 IEEE 72nd Vehicular Technology Conference-Fall. IEEE, 2010. p. 1-5. ZHOU, Binbin, et al. Adaptive traffic light control in wireless sensor network-based intelligent transportation system. In: 2010 IEEE 72nd Vehicular Technology Conference-Fall. IEEE, 2010. p. 1-5. 